\title{
Correction to: PEGylation of graphene/iron oxide nanocomposite: assessment of release of doxorubicin, magnetically targeted drug delivery and photothermal therapy
}

\author{
Marzieh Ramezani Farani ${ }^{1}$ - Parissa Khadiv-Parsi ${ }^{1}$. Gholam Hossein Riazi ${ }^{2} \cdot$ Mehdi Shafiee Ardestani $^{3}$ (1) . \\ Hamidreza Saligheh $\operatorname{Rad}^{4,5}$
}

Published online: 9 April 2020

(c) King Abdulaziz City for Science and Technology 2020

\section{Correction to: Applied Nanoscience (2020) 10:1205-1217 https://doi.org/10.1007/s13204-020-01255-8}

The article PEGylation of graphene/iron oxide nanocomposite: assessment of release of doxorubicin, magnetically targeted drug delivery and photothermal therapy, written by Marzieh Ramezani Farani, Parissa Khadiv-Parsi, Gholam Hossein Riazi, Mehdi Shafiee Ardestani, Hamidreza Saligheh Rad, was originally published electronically on the publisher's internet portal (currently SpringerLink) on 30 January 2020 with open access. With the author(s)' decision to step back from Open Choice, the copyright of the article changed on 29 March 2020 to (c) King Abdulaziz City for Science and Technology 2020 and the article is forthwith distributed under the terms of copyright.

The original article can be found online at https://doi.org/10.1007/ s13204-020-01255-8.

Parissa Khadiv-Parsi

kparsi@ut.ac.ir

$\triangle$ Mehdi Shafiee Ardestani

shafieeardestani@tums.ac.ir

1 School of Chemical Engineering, University College of Engineering, University of Tehran, 4563-11155 Tehran, Iran

2 Institute of Biophysics and Biochemistry, University of Tehran, 1417614411 Tehran, Iran

3 Department of Radio-Pharmacy, Faculty of Pharmacy, Tehran University of Medical Sciences, 1417614411 Tehran, Iran

4 Quantitative Medical Imaging Systems Group, Research Center for Molecular and Cellular Imaging, Tehran University of Medical Sciences, 1417613151 Tehran, Iran

5 Medical Physics and Biomedical Engineering Department, Tehran University of Medical Sciences, 1417613151 Tehran, Iran 